\title{
FDG-PET/CT and diffusion-weighted imaging for resected lung cancer: correlation of maximum standardized uptake value and apparent diffusion coefficient value with prognostic factors
}

\author{
Katsuo Usuda ${ }^{1}$ (1) Aika Funasaki ${ }^{1} \cdot$ Atsushi Sekimura $^{1} \cdot$ Nozomu Motono $^{1} \cdot$ Munetaka Matoba $^{2} \cdot$ Mariko Doai $^{2}$. \\ Sohsuke Yamada ${ }^{3} \cdot$ Yoshimichi Ueda $^{4} \cdot$ Hidetaka Uramoto $^{1}$
}

Received: 18 March 2018 / Accepted: 6 April 2018 / Published online: 9 April 2018

(c) The Author(s) 2018

\begin{abstract}
Diffusion-weighted magnetic resonance imaging (DWI) is useful for detecting malignant tumors and the assessment of lymph nodes, as FDG-PET/CT is. But it is not clear how DWI influences the prognosis of lung cancer patients. The focus of this study is to evaluate the correlations between maximum standardized uptake value (SUVmax) of FDG-PET/CT and apparent diffusion coefficient (ADC) value of DWI with known prognostic factors in resected lung cancer. A total of 227 patients with resected lung cancers were enrolled in this study. FEG-PET/CT and DWI were performed in each patient before surgery. There were 168 patients with adenocarcinoma, 44 patients with squamous cell carcinoma, and 15 patients with other cell types. SUVmax was a factor that was correlated to $\mathrm{T}$ factor, $\mathrm{N}$ factor, or cell differentiation. ADC of lung cancer was a factor that was not correlated to $\mathrm{T}$ factor, or $\mathrm{N}$ factor. There was a significantly weak inverse relationship between SUVmax and ADC (Correlation coefficient $r=-0.227$ ). In analysis of survival, there were significant differences between the categories of sex, age, pT factor, pN factor, cell differentiation, cell type, and SUVmax. Univariate analysis revealed that SUVmax, $\mathrm{pN}$ factor, age, cell differentiation, cell type, sex, and $\mathrm{pT}$ factor were significant factors. Multivariate analysis revealed that SUVmax and $\mathrm{pN}$ factor were independent significant prognostic factors. SUVmax was a significant prognostic factor that is correlated to $\mathrm{T}$ factor, $\mathrm{N}$ factor, or cell differentiation, but ADC was not. SUVmax may be more useful for predicting the prognosis of lung cancer than ADC values.
\end{abstract}

Keywords Lung cancer · Diagnosis · Diffusion-weighted magnetic resonance imaging (DWI) $\cdot$ Magnetic resonance imaging (MRI) $\cdot$ Positron emission tomography (PET) $\cdot$ Prognostic factor

\author{
Katsuo Usuda \\ usuda@kanazawa-med.ac.jp \\ Aika Funasaki \\ aicarby@kanazawa-med.ac.jp \\ Atsushi Sekimura \\ a24seki@kanazawa-med.ac.jp \\ Nozomu Motono \\ motono@kanazawa-med.ac.jp \\ Munetaka Matoba \\ m-matoba@kanazawa-med.ac.jp \\ Mariko Doai \\ doaimari@kanazawa-med.ac.jp \\ Sohsuke Yamada \\ sohsuke@kanazawa-med.ac.jp
}

\author{
Yoshimichi Ueda \\ z-ueda@kanazawa-med.ac.jp \\ Hidetaka Uramoto \\ hidetaka@kanazawa-med.ac.jp \\ 1 Department of Thoracic Surgery, Kanazawa Medical \\ University, 1-1 Daigaku, Uchinada, Ishikawa 920-0293, \\ Japan \\ 2 Department of Radiology, Kanazawa Medical University, \\ Uchinada, Japan \\ 3 Department of Pathology and Laboratory Medicine, \\ Kanazawa Medical University, Uchinada, Japan \\ 4 Department of Pathophysiological and Experimental \\ Pathology, Kanazawa Medical University, Uchinada, Japan
}




\begin{tabular}{ll}
\multicolumn{2}{l}{ Abbreviations } \\
FDG-PET & $\begin{array}{l}\text { Positron emission tomography with } \\
\text { 18-fluoro-2-deoxy-glucose }\end{array}$ \\
DWI & $\begin{array}{l}\text { Diffusion-weighted magnetic resonance } \\
\text { imaging }\end{array}$ \\
SUVmax & $\begin{array}{l}\text { Maximum standardized uptake value } \\
\text { MRI }\end{array}$ \\
Magnetic resonance imaging \\
ADC & Apparent diffusion coefficient \\
ROI & Large cell neuroendocrine carcinoma \\
OCV & $\begin{array}{l}\text { Region of interest } \\
\text { Optimal cutoff value }\end{array}$ \\
SPAIR & Spectral attenuated inversion recovery
\end{tabular}

\section{Introduction}

Lung cancer is a heterogeneous cancer that has various patterns of progression and treatment responses. Positron emission tomography with 18-fluoro-2-deoxy-glucose (FDGPET) has been widely adopted as the imaging method of choice in tumor staging. The maximum standardized uptake value (SUVmax) is a parameter of glucose uptake and usually indicates how aggressive the cancer is. FDG-PET/CT has helped differentiate malignant from benign pulmonary nodules [1]. However, FDG-PET/CT can produce false-negative results for well-differentiated pulmonary adenocarcinoma [2], or small volumes of metabolically active tumors [3], and false-positive results for inflammatory nodules [4].

For the last two decades, magnetic resonance imaging (MRI) in lung cancer staging has been limitedly used in mediastinum invasion or chest wall invasion of lung cancer after Webb et al. [5] of the Radiologic Diagnostic Oncology Group published results in 1991. Diffusion-weighted magnetic resonance imaging (DWI) has been applied to detect the restricted diffusion of water molecules. The principals of DWI utilize the random motion of water molecules in biologic tissue [6]. Apparent diffusion coefficient (ADC) value is a quantitative parameter of the diffusion of water molecules in biological tissues, and is usually significantly lower in malignant tumors compared with normal tissue or benign lesions [7]. The MR signal intensity of pulmonary cancer nodules was significantly higher than that of benign lesions [8]. A meta-analysis has shown that DWI can be used to differentiate malignant from benign pulmonary lesions [9]. Two articles of meta-analysis reported that DWI was effective for the evaluation of $\mathrm{N}$ factor of lung cancer [10, 11]. Peerlings et al. [10] reported high diagnostic capability of DWI for nodal assessment in the non-small cell lung cancer: The sensitivity was 0.87 and the specificity 0.88 . DWI can distinguish benign from malignant lesions in the lung $[9,12]$, in the thorax [13], in the prostate [14], in the breast [15], and in the liver [16].
There were two articles which compared diagnostic capability of DWI with that of FDG-PET/CT for pulmonary nodules and masses $[12,17]$ : The sensitivity and the accuracy of DWI were significantly higher [12], or the sensitivity of DWI was significantly higher [17] than those of FDG-PET/ CT. DWI was reported to be superior to FDG-PET in the detection of primary lesions and the nodal assessment of non-small cell lung cancers [18].

The SUVmax of FDG-PET was reported to be a significant prognostic factor of lung cancer [19]. To our knowledge, there are no articles that have combined FDG-PET/ $\mathrm{CT}$ and DWI to evaluate the prognostic value of preoperative SUVmax and ADC in lung cancers. The focus of this study is to determine the correlation of SUVmax of FDG-PET/CT and ADC value of DWI with known prognostic factors and to evaluate their prognostic values.

\section{Materials and methods}

\section{Eligibility}

The study protocol for examining DWI and FDG-PET/CT in patients with lung cancer was approved by the ethical committee of Kanazawa Medical University (the approval number: No. 189). Written informed consents for MRI, PETCT and a pathological examination of resected materials were obtained from each patient after discussing the risks and benefits of the examinations with their surgeons.

\section{Patients}

Two-hundred and twenty-seven patients with primary lung cancer were enrolled in this study. They underwent DWI and PET-CT examination before pulmonary resection with nodal dissection from May 2009 to February 2014. None of the patients had received prior treatment. One-hundred and thirty-four patients were male and 93 were female. Their mean age was 68 years old (range 37-85).

Cell type, cell differentiation, pathological $\mathrm{N}$ factor, and the size of the tumor were determined by reviewing the pathology reports. There were 168 adenocarcinomas, 44 squamous cell carcinomas, 5 small cell carcinomas, 3 large cell neuroendocrine carcinoma (LCNEC), 3 large cell carcinomas, and 3 carcinomas of other cell types. TNM classification and the lymph node stations of lung cancer were classified according to the new definition of UICC 7 [20]. There were 113 pathological Stage IA (pStage IA), 49 pStage IB, 20 pStageIIA, 14 pStage IIB, 25 pStage IIIA, 1 pStage IIIB, and 5 pStage IV. There were 77 pathological T1a (pT1a) carcinomas, 42 pT1b carcinomas, 65 pT2a carcinomas, 13 pT2b carcinomas, 25 pT3 carcinomas, and 
5 pT4 carcinomas. There were 180 pathological pN0 (pN0) carcinomas, $30 \mathrm{pN} 1$ carcinomas, and $17 \mathrm{pN} 2$ carcinomas.

\section{PET-CT}

FDG-PET scanning was performed with a dedicated PET camera (SIEMENS Biograph Sensation 16, Erlangen Germany) before surgery. All patients fasted for $6 \mathrm{~h}$ before scanning. The dose of ${ }^{18} \mathrm{~F}$-FDG administered was $3.7 \mathrm{MBq} / \mathrm{Kg}$ of body weight. After a 60 -min uptake period, an emission scan was acquired for 3 min per bed position and a whole-body scan was performed according to the height of each patient. After image reconstruction, a two-dimensional (2D) round region of interest (ROI) was drawn on a slice after visual detection of the highest count on the fused CT image by a radiologist (N.W.) with 29 years of radioisotope scintigraphy and PET-CT experience who was unaware of the patients' clinical data. For the lesions with negative or faintly positive PET findings, the ROI was drawn on the fusion image with the corresponding CT. From those ROI, the maximum standardized uptake value (SUVmax) was calculated. The radiologist (N.W.) and one pulmonologist (K.U.) with 28 years of experience evaluated the FDG-PET data. They eventually reached at the same consensus. The optimal cutoff value (OCV) of SUVmax for diagnosing malignancy in PET-CT was determined to be 4.45 using the receiver operating characteristics curve as previously reported [21].

\section{MR imaging}

All MR images were created with a $1.5 \mathrm{~T}$ superconducting magnetic scanner (Magnetom Avanto; Siemens, Erlangen, Germany) with two anterior six-channel body phasedarray coils and two posterior spinal clusters (six channels each). The conventional MR images consisted of a coronal T1-weighted spin-echo sequence and coronal and axial T2-weighted fast spin-echo sequences. DWIs using a singleshot echo-planar method were performed with slice thickness of $6 \mathrm{~mm}$ under SPAIR (spectral attenuated inversion recovery) with respiratory triggered scan with the following parameter: TR/TE/flip angle, 3000-4500/65/90; diffusion gradient encoding in three orthogonal directions; $b$ value $=0$ and $800 \mathrm{~s} / \mathrm{mm}^{2}$; field of view, $350 \mathrm{~mm}$; and matrix size, $128 \times 128$. After image reconstruction, a two-dimensional (2D) round or elliptical region of interest (ROI) was drawn on the lesion which was detected visually on the ADC map with reference to $\mathrm{T} 2$-weighted or CT image by a radiologist (M.D.) with 25 years of MRI experience who was unaware of the patients' clinical data. Areas with necrosis were excluded from the ADC measurement. The procedure was repeated three times, and the minimum ADC value was obtained. The radiologist (M.D.) and one pulmonologist (K.U.) with 28 years of experience evaluated the MRI data.
They eventually reached at the same consensus. The OCV of ADC for diagnosing malignancy in DWI was determined to be $1.70 \times 10^{-3} \mathrm{~mm}^{2} / \mathrm{s}$ using the receiver operating characteristics curve as previously reported [21].

\section{Statistical analysis}

The data are expressed as the mean \pm standard deviation. A two-tailed Student t test was used for comparison of ADC values or SUVmax in several prognostic factors. The Kaplan-Meier method was used to calculate the survival rate using death from any cause with a 95\% confidence interval (CI), and the log-rank test was used to compare the survival curves. A Cox proportional hazard model was used for the univariate and multivariate survival analyses. The statistical analyses were performed using the computer software program StatView for Windows (Version 5.0; SAS Institute Inc. Cary, NC, USA). A $P$ value of $<0.05$ was considered statistically significant.

\section{Results}

Radiological images of a lung cancer (adenocarcinoma) is presented in Fig. 1. There was a significantly weak inverse relationship between SUVmax and ADC (Correlation coefficient $r=-0.227, P=0.0006$; Fig. 2).

Correlations between the SUVmax and several prognostic factors are presented in Fig. 3. There were a significant correlation between the SUVmax and the pT factor (Fig. 3b). The SUVmax of pN0, pN1, or pN2 lung cancer was $5.13 \pm 4.99,12.67 \pm 7.27$, or $10.21 \pm 6.11$, respectively. The SUVmax of pN0 lung cancer was significantly lower than that of $\mathrm{pN} 1$ or $\mathrm{pN} 2$ lung cancer $(P<0.0001$; Fig. 3c). There was a significant correlation between the SUVmax and cell differentiation (Fig. 3d). The SUVmax was a factor that is correlated to $\mathrm{T}$ factor, $\mathrm{N}$ factor, and cell differentiation.

Correlations between ADC and several prognostic factors are presented in Fig. 4. There was no correlation between ADC and the several prognostic factors (Fig. 4a, b, d). $\mathrm{ADC}$ of $\mathrm{pN} 0, \mathrm{pN} 1$, or $\mathrm{pN} 2$ lung cancer was $1.29 \pm 0.34$, $1.24 \pm 0.33,1.17 \pm 0.20 \times 10^{-3} \mathrm{~mm}^{2} / \mathrm{s}$, respectively (Fig. $4 \mathrm{c}$ ). $\mathrm{ADC}$ of lung cancer is a factor that is not correlated to $\mathrm{T}$ factor, or $\mathrm{N}$ factor.

Survival curves by several factors are presented in Fig. 5 . Five-year survival rate (0.89) of female patients was significantly higher than that $(0.67)$ of male patients $(P=0.0037)$. Five-year survival rate $(0.85)$ of patients under 70 years old was significantly higher than that $(0.65)$ of patients 70 years old or older $(P=0.0160)$. For survival rates by pT factor, there were significant differences among them $(P<0.0001$; Fig. 5a). For survival rates by $\mathrm{pN}$ factor, 5-year survival rates of $\mathrm{pN} 0$, $\mathrm{pN} 1$, or $\mathrm{pN} 2$ lung cancer were $0.82,0.70$, or 

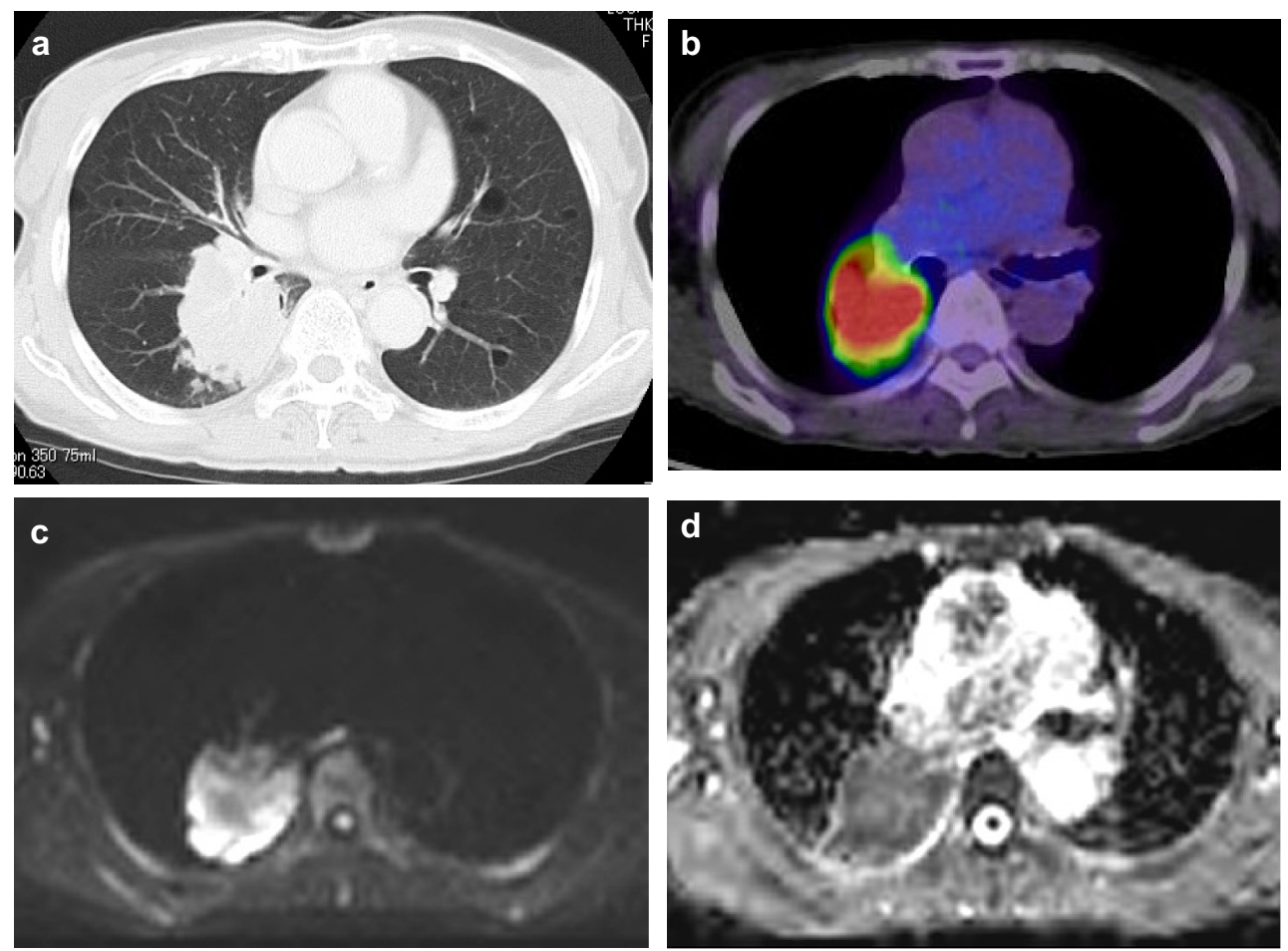

Fig. 1 Adenocarcinoma a CT, b PET-CT, SUVmax 7.79, c DWI, and d ADCmap, ADC $1.165 \times 10^{-3} \mathrm{~mm}^{2} / \mathrm{s}$

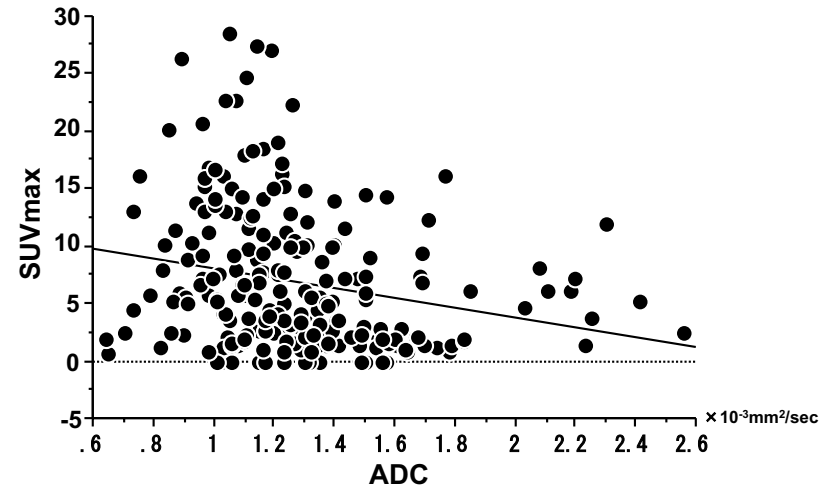

Fig. 2 Correlation between SUVmax and ADC of lung cancer. SUVmax $=12.40-4.34 \times$ ADC. Correlation coefficient $r=-0.227$, $P=0.0006$

0.24 , respectively. There were significant differences among them $(P<0.0001 ;$ Fig. $5 b)$. For survival rates by cell differentiation, there were significant differences among them $(P=0.0054)$. For survival rates by cell type, 5 -year survival rate $(0.80)$ of patients with adenocarcinomas was significantly higher than that $(0.62)$ of patients with other cell types $(P=0.0003)$. The SUVmax was divided into two groups by the mean value of 6.50 . The 5-year survival rate $(0.85)$ of patients in the SUVmax low group with an SUVmax under 6.50 was significantly higher than that $(0.59)$ of patients in the SUVmax high group with an SUVmax of 6.50 or more $(P<0.0001)$. (Figure $5 \mathrm{c})$. ADC was divided into two groups by the mean value of $1.27 \times 10^{-3} \mathrm{~mm}^{2} / \mathrm{s}$. The 5 -year survival rate (0.80) of patients in the ADC low group with an ADC under $1.27 \times 10^{-3} \mathrm{~mm}^{2} / \mathrm{s}$ was not higher than that (0.70) of patients in the ADC high group with an ADC of $1.27 \times 10^{-3} \mathrm{~mm}^{2} / \mathrm{s}$ or more $(P=0.768 ;$ Fig. $5 \mathrm{~d})$.

Univariate analysis using a Cox proportional hazard model revealed that SUVmax, pN factor, age, cell differentiation, cell type, sex, and pT factor were significant (Table 1). But ADC was not a significant factor. The multivariate analysis of factors influencing survival by a Cox proportional hazard model revealed that independent significant prognostic factors were SUVmax $(P=0.0202)$ and $\mathrm{pN}$ factors $(P=0.0353)$, and ADC was not $(P=0.0581$; Table 2$)$.

\section{Discussion}

Our main finding was that SUVmax of lung cancer has a stronger relationship with known prognostic factors and may be more useful for predicting the prognosis of lung cancer than ADC values of lung cancer. Although ADC of DWI is useful to distinguish benign from malignant lesions in the lung, ADC itself is not a significant prognostic factor in lung cancer and is not related to known prognostic factors. The difference found in prognostic significance can be explained 

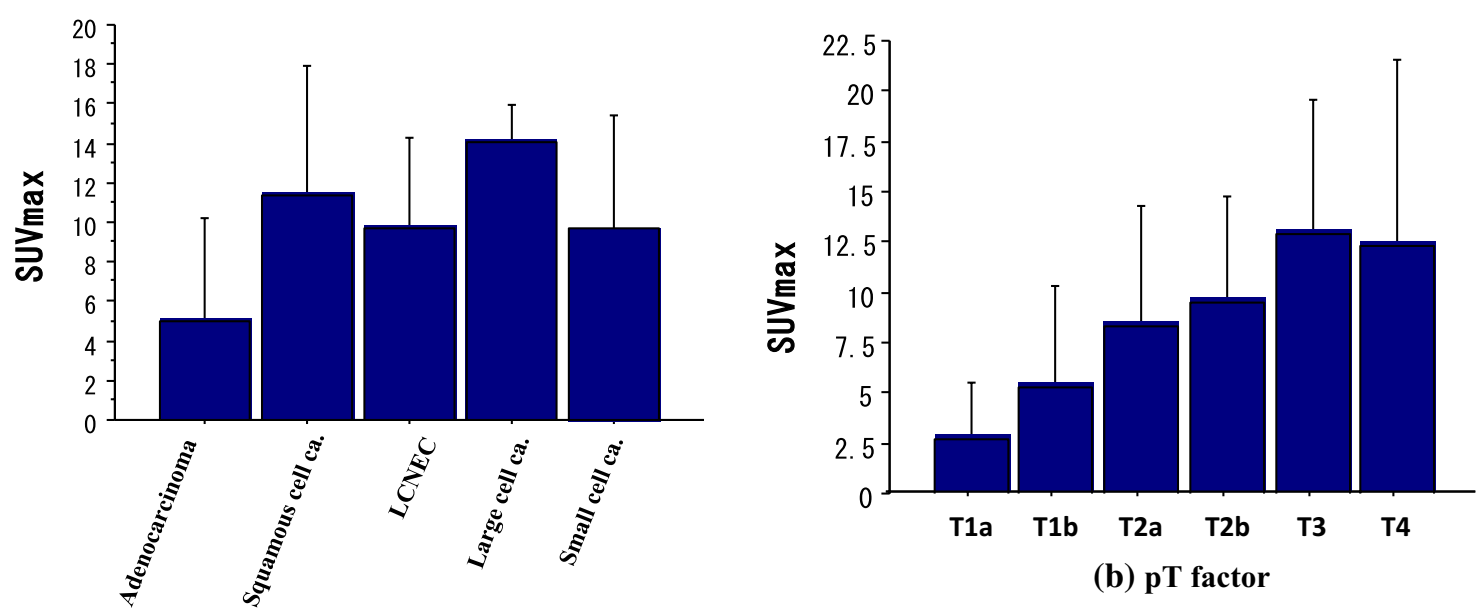

(a) Cell types

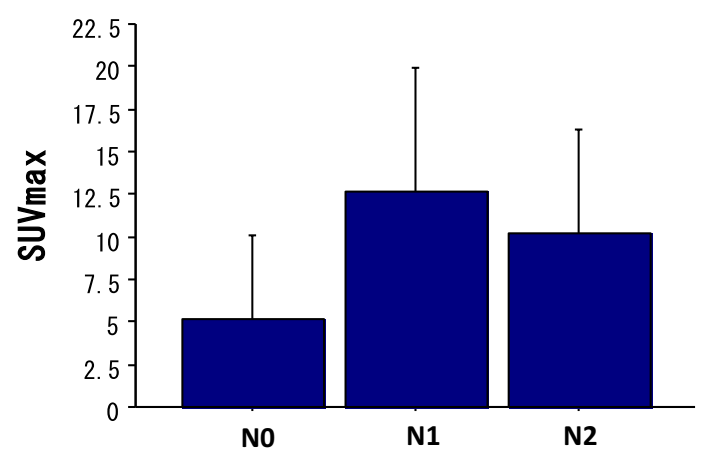

(c) $\mathrm{pN}$ factor

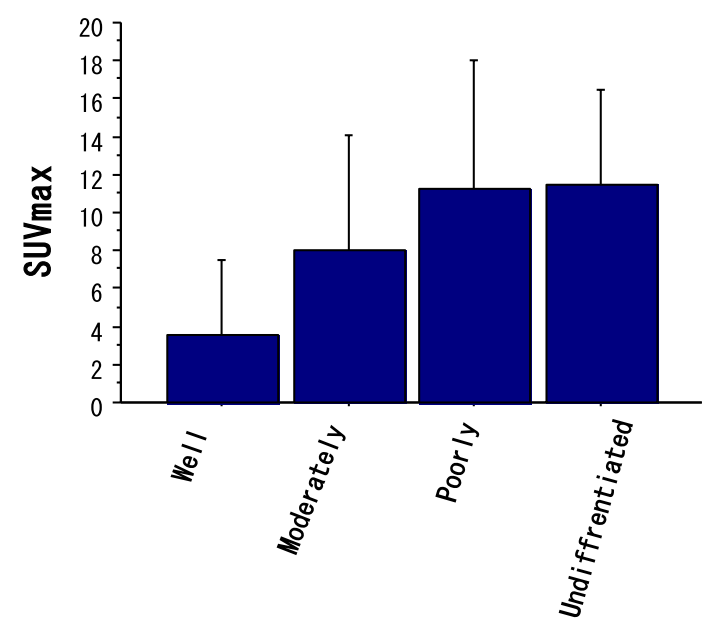

(d) Cell differentiation

Fig. 3 Correlation between SUVmax and several prognostic factors. There were a significant correlation between SUVmax and T factor/cell differentiation

by the fact that FDG-PET provides quantitative information regarding cellular glucose metabolism which is associated to tumor aggressiveness, while DWI provides quantitative information regarding tissue cellularity and the diffusion of water molecules which are not necessarily associated to tumor aggressiveness. In our study, there was a significantly weak inverse relationship between ADC and SUVmax. It may mean that the higher SUVmax of lung cancer is, the lower the corresponding ADC is. Although SUVmax and ADC represent different aspects of the biologic features of the tumor, SUVmax showing metabolic activity may be correlated to ADC showing tumor cellularity and diffusion of water.
In breast cancer, Karan et al. [22] and Kitajima et al. [23] reported that SUVmax was significantly associated with known prognostic factors such as tumor size, histological grade, lymph node status, estrogen receptor status, human epidermal growth factor receptor 2 status, whereas ADC values were not, which concluded that SUVmax may be valuable for predicting the prognosis of breast cancer. This result is similar to our data. On the other hand, Nakajo et al. [24] and Choi et al. [25] mentioned that SUVmax and ADC correlated with several pathological prognostic factors and both indexes may have the same potential for predicting the prognosis of breast cancer. DWI-MRI and FDG-PET/ CT have their own advantages [26]. Gallivanone et al. [27] 

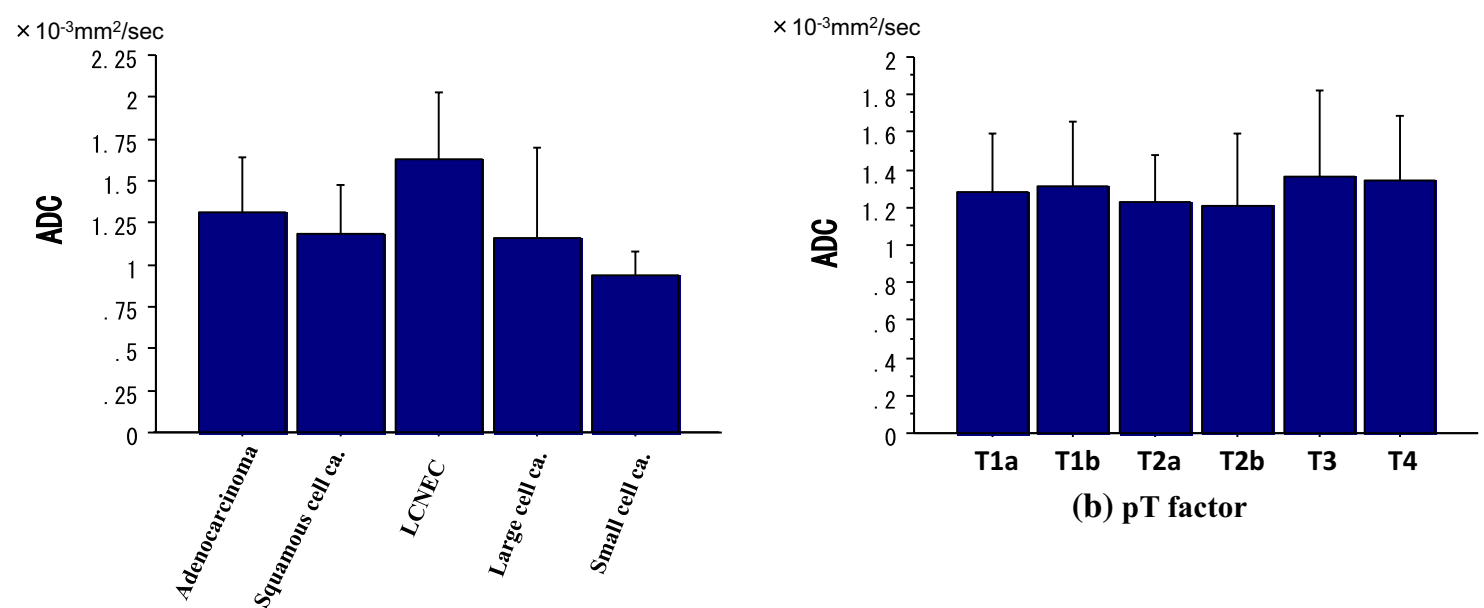

(b) pT factor

(a) Cell types

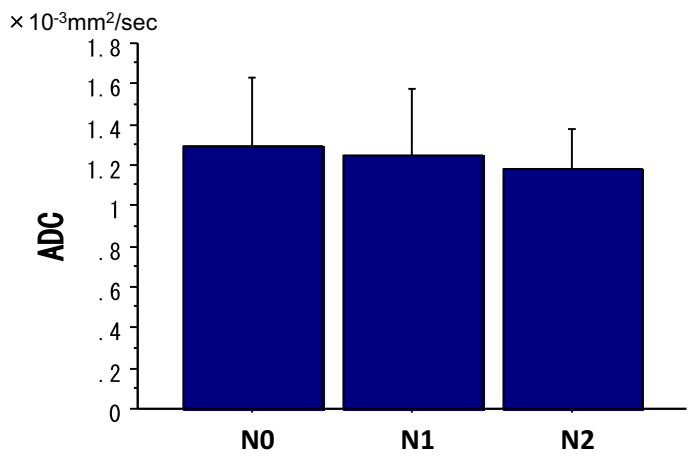

(c) pNfactor

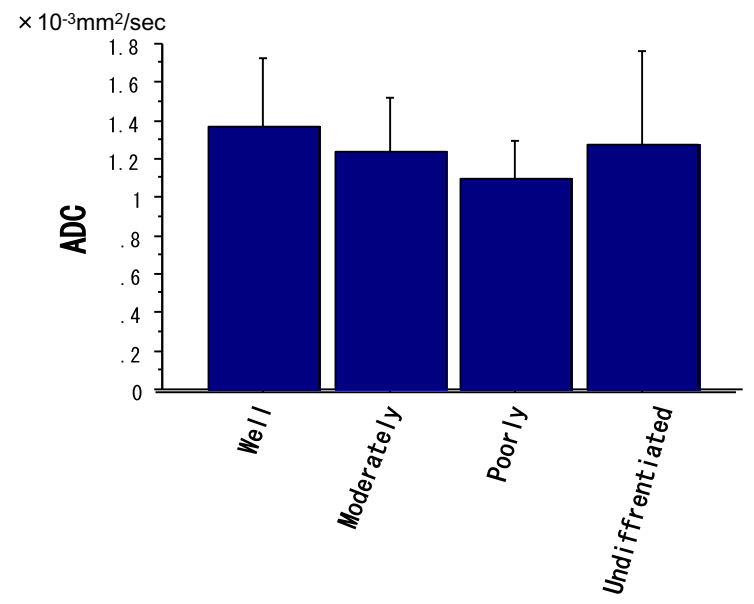

(d) Cell differentiation

Fig. 4 Correlation between ADC and several prognostic factors. There was no correlation between ADC and the several prognostic factors

reported that FDG-PET predicts patient prognosis and DWI predicts response to neoadjuvant chemotherapy, and both examinations provide useful complementary information for biological characterization and neoadjuvant chemotherapy response prediction in breast cancer.

We have to keep in mind that there are two important limitations in this study. First, it had a retrospective study and was conducted at a single institution, which would have unavoidably introduced selection bias. Second, our ADC measurements were repeated three times and the minimum ADC value was obtained. This ADC may not be fully representative for the whole tumor. There is no consensus in the literature concerning the optimal DWI techniques and image analysis procedure, including ROI size and placement.

Concerning survival of patients with locally advanced non-small cell lung cancer, staging with FDG-PET/CT was reported to be superior to conventional staging methods [28]. DWI was reported to have better potential than FDG-PET/CT for prediction of tumor response to therapy in non-small cell lung cancer patients before chemoradiotherapy [29]. Further studies are necessary to evaluate the performance of FDG-PET/CT and DWI for treatment and survival of lung cancer patients. 
(a)

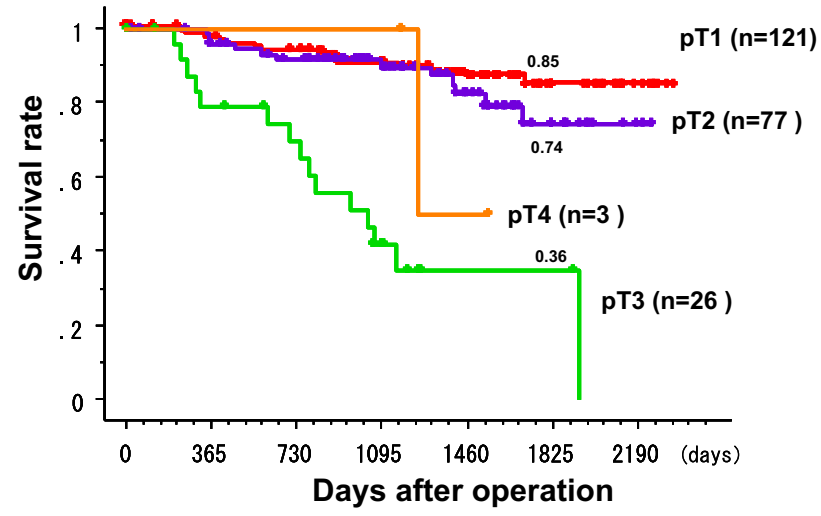

(c)

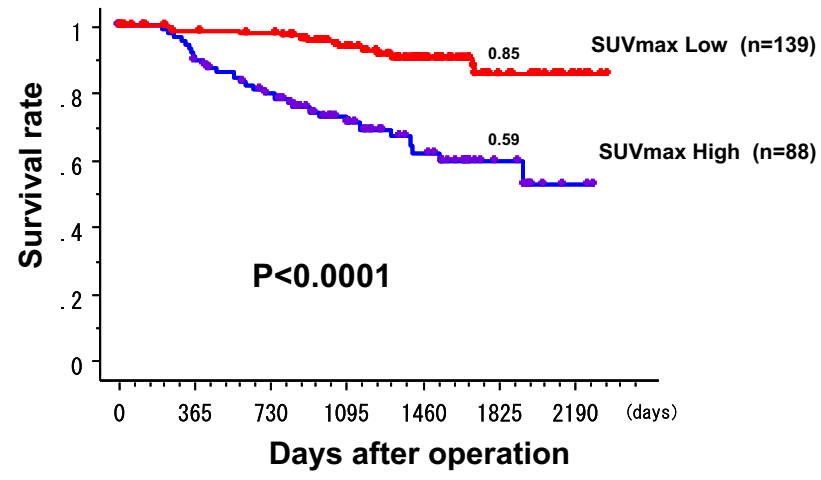

Fig. 5 a Survival rates by pT factor. There were significant differences among them $(P<0.0001)$. b Survival rates by $\mathrm{pN}$ factor. There were significant differences among them $(P<0.0001)$. c Survival rates by SUVmax. SUVmax was divided into two groups by the mean value of 6.50 . The 5 -year survival rate $(0.85)$ of patients in the SUVmax low group where the SUVmax was under 6.50 was significantly higher than that $(0.59)$ of patients in the SUVmax high group (b)

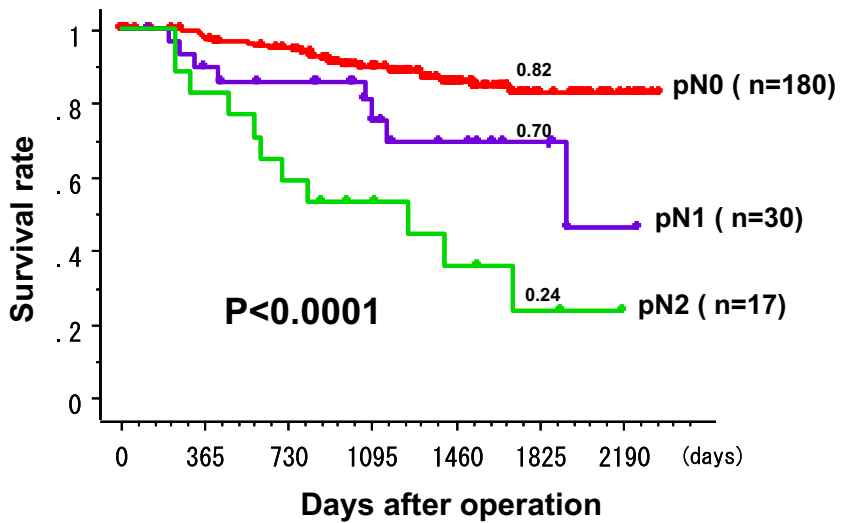

(d)

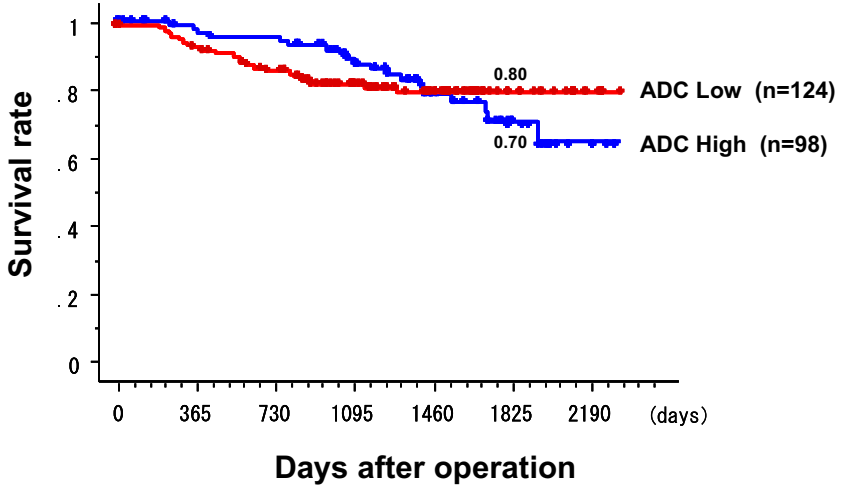

where the SUVmax was 6.50 or higher $(P<0.0001)$. d Survival rates by ADC. ADC was divided into two groups by the mean value of $1.27 \times 10^{-3} \mathrm{~mm}^{2} / \mathrm{s}$. The 5 -year survival rate $(0.80)$ of patients in the ADC low group where the ADC was under $1.27 \times 10^{-3} \mathrm{~mm}^{2} / \mathrm{s}$ was not higher than that $(0.70)$ of patients in the ADC high group where the ADC was $1.27 \times 10^{-3} \mathrm{~mm}^{2} / \mathrm{s}$ or higher $(P=0.768)$

Table 1 Univariate analysis

\begin{tabular}{lllllc}
\hline Factor & & Hazard ratio & $95 \%$ CI & & $P$ value \\
\hline SUVmax & Low/high & 4.485 & 2.325 & 8.652 & $<0.0001$ \\
pN factor & pN0/pN1-2 & 0.257 & 0.14 & 0.473 & $<0.0001$ \\
ADC & Low/high & 1.108 & 0.599 & 2.05 & 0.7426 \\
Age & Under 70 years old/70 years old or more & 2.086 & 1.112 & 3.914 & 0.0219 \\
Cell differentiation & Well/moderately $\sim$ undifferentiated & 0.329 & 0.165 & 0.656 & 0.0016 \\
Cell type & Adenocarcinoma/other cell types & 0.352 & 0.191 & 0.646 & 0.0008 \\
Sex & Female/male & 0.354 & 0.169 & 0.743 & 0.006 \\
pT factor & pT1/pT2-4 & 0.381 & 0.2 & 0.724 & 0.0033 \\
\hline
\end{tabular}


Table 2 Multivariate analysis

\begin{tabular}{llllll}
\hline Factor & & Hazard ratio & 95\% CI & & P value \\
\hline SUVmax & Low/high & 2.728 & 1.17 & 6.363 & 0.0202 \\
pN factor & pN0/pN1-2 & 0.469 & 0.231 & 0.949 & 0.0353 \\
ADC & Low/high & 1.939 & 0.977 & 0.3846 & 0.0581 \\
Age & Under 70 years old/70 years old or more & 1.714 & 0.888 & 3.308 & 0.108 \\
Cell differentiation & Well/moderately $\sim$ undifferentiated & 0.611 & 0.266 & 1.399 & 0.2436 \\
Cell type & Adenocarcinoma/other cell types & 0.698 & 0.335 & 1.454 & 0.3369 \\
Sex & Female/male & 0.687 & 0.306 & 1.541 & 0.3625 \\
pT factor & pT1/pT2-4 & 0.997 & 0.449 & 2.212 & 0.9934 \\
\hline
\end{tabular}

\section{Conclusion}

SUVmax is a significant prognostic factor that is correlated to known prognostic factors. But ADC of DWI is not correlated to these factors and not a significant prognostic factor. SUV max may be more useful for predicting the prognosis of lung cancer than ADC values.

Acknowledgements We are grateful to Mr. Keiya Hirata of the MRI Center, Kanazawa Medical University, for technical assistance.

Funding This study was supported partly by a Grant-in-Aid for Scientific Research from the Ministry of Education, Culture, Sports, Science, and Technology, Japan (Grant Number: 16K10694) and by 2017 Grantin-Aid of the Magnetic Health Science Foundation, Japan.

\section{Compliance with ethical standards}

Conflict of interest The authors declared that they have no conflict of interest.

Open Access This article is distributed under the terms of the Creative Commons Attribution 4.0 International License (http://creativeco mmons.org/licenses/by/4.0/), which permits unrestricted use, distribution, and reproduction in any medium, provided you give appropriate credit to the original author(s) and the source, provide a link to the Creative Commons license, and indicate if changes were made.

\section{References}

1. Could MK, Maclean CC, Kuschner WG, et al. Accuracy of positron emission tomography for diagnosis of pulmonary nodules and mass lesions. A meta-analysis. JAMA. 2001;285:914-24.

2. Cheran SK, Nielsen ND, Patz EF. False-negative findings for primary lung tumors on FDG positron emission tomography. Staging and prognostic implications. AJR. 2004;182:1129-32.

3. Satoh Y, Ichikawa T, Motosugi U, et al. Diagnosis of peritoneal dissemination. Comparison of 18F-DDG PET/CT, diffusion-weighted MRI, and contrast-enhanced MDCT. AJR. 2011;196:447-53.

4. Goo JM, Im JG, Do KH, et al. Pulmonary tuberculoma evaluated by means of FDG PET. Findings in 10 cases. Radiology. 2000;216:117-21.

5. Webb WR, Gatsonis C, Zerhouni EA, et al. CT and MR imaging in staging non-small cell bronchogenic carcinoma. Report of the radiologic diagnostic oncology group. Radiology. 1991;178:705-13.

6. Le Bihan D, Breton E, Lallemand D, et al. Separation of diffusion and perfusion in intravoxel incoherent motion MR imaging. Radiology. 1988;168:497-505.

7. Szafer A, Zhong J, Gore JC. Theoretical model for water diffusion in tissues. Magn Reson Med. 1995;33:697-712.

8. Uto T, Takehara Y, Nakamura Y, et al. Higher sensitivity and specificity for diffusion-weighted imaging of malignant lung lesions without apparent diffusion coefficient quantification. Radiology. 2009;252:247-54.

9. Wu LM, Xu JR, Hua J, et al. Can diffusion-weighted imaging be used as a reliable sequence in the detection of malignant pulmonary nodules and masses? Magn Reson Imaging. 2013;31:235-46.

10. Peerlings J, Troost EG, Nelemans PJ, et al. The diagnostic value of MR Imaging in determining the lymph node status of patients with non-small cell lung cancer. A meta-analysis. Radiology. 2016;281:86-98.

11. Shen G, Hu S, Deng H, et al. Performance of DWI in the nodal characterization and assessment of lung cancer: a meta-analysis. Am J Roentgenol. 2016;206:283-90.

12. Mori T, Nomori H, Ikeda K, et al. Diffusion-weighted magnetic resonance imaging for diagnosing malignant pulmonary nodules/ masses. Comparison with positron emission tomography. J Thorac Oncol. 2008;3:358-64.

13. Tondo F, Saponaro A, Stecco A, et al. Role of diffusion-weighted imaging in the differential diagnosis of benign and malignant lesions of the chest-mediastinum. Radiol Med. 2011;116:720-33.

14. Yamamura J, Salomon G, Buchert R, et al. Magnetic resonance imaging of prostate cancer. Diffusion-weighted imaging in comparison with sextant biopsy. J Comput Assist Tomogr. 2011;35:223-8.

15. Fornasa F, Pinali L, Gasparini A, et al. Diffusion-weighted magnetic resonance imaging in focal breast lesions. Analysis of 78 cases with pathological correlation. Radiol Med. 2011;116:264-75.

16. Koike N, Cho A, Nasu K, et al. Role of diffusion-weighted magnetic resonance imaging in the differential diagnosis of focal hepatic lesions. World J Gastroenterol. 2009;15:5805-12.

17. Usuda K, Sagawa M, Motono N, et al. Diagnostic performance of diffusion weighted imaging of malignant and benign pulmonary nodules and masses: comparison with positron emission tomography. Asian Pac J Cancer Prev. 2014;15:4629-35.

18. Usuda K, Zhao XT, Sagawa M, et al. Diffusion-weighted imaging is superior to PET in the detection and nodal assessment of lung cancers. Ann Thorac Surg. 2011;91:1689-95.

19. Borst GR, Belderbos JS, Boellaard R, et al. Standardised FDG uptake. a prognostic factor for inoperable non-small cell lung cancer. Eur J Cancer. 2005;41:1533-41. 
20. International Union Against Cancer. TNM classification of malignant tumours. 7th ed. New York: Wiley-Liss; 2009. p. $138-46$.

21. Usuda K, Zhao XT, Sagawa M, et al. Diffusion-weighted imaging is superior to PET in the detection and nodal assessment of lung cancers. Ann Thorac Surg. 2011;91:1689-95.

22. Karan B, Pourbagher A, Torun N. Diffusion-weighted imaging and 18 F-fluorodeoxyglucose positron emission tomography/computed tomography in breast cancer: correlation of the apparent diffusion coefficient and maximum standardized uptake values with prognostic factors. J Magn Reson Imaging. 2016;43:1434-44.

23. Kitajima K, Yamano T, Fukushima K, et al. Correlation of the SUVmax of FDG-PET and ADC values of diffusion-weighted MR imaging with pathologic prognostic factors in breast carcinoma. Eur J Radiol. 2016;85:943-9.

24. Nakajo M, Kajiya Y, Kaneko T, et al. FDG PET/CT and diffusionweighted imaging for breast cancer: prognostic value of maximum standardized uptake values and apparent diffusion coefficient values of the primary lesion. Eur J Nucl Med Mol Imaging. 2010;37:2011-20.

25. Choi BB, Kim SH, Park CS, et al. Correlation of prognostic factors of invasive lobular carcinoma with ADC Value of DWI and SUVMax of FDG-PET. Chonnam Med J. 2017;53:133-9.
26. Dubreuil J, Tordo J, Rubello D, et al. Diffusion-weighted MRI and 18F-FDG-PET/CT imaging: competition or synergy as diagnostic methods to manage sarcoma of the uterus? A systematic review of the literature. Nucl Med Commun. 2017;38:84-90.

27. Gallivanone F, Panzeri MM, Canevari C, et al. Biomarkers from in vivo molecular imaging of breast cancer: pretreatment $18 \mathrm{~F}$ FDG PET predicts patient prognosis, and pretreatment DWI-MR predicts response to neoadjuvant chemotherapy. MAGMA (Magn Reson Mater Phys Biol Med). 2017;30:359-73.

28. Mutlu H, Buyukcelik A, Erden A, et al. Staging with PET-CT in patients with locally advanced non small cell lung cancer is superior to conventional staging methods in terms of survival. Asian Pac J Cancer Prev. 2013;14:3743-6.

29. Ohno Y, Koyama H, Yoshikawa T, Matsumoto K, Aoyama N, Onishi Y, Sugimura K, et al. Diffusion-weighted MRI versus 18F-FDG PET/CT. Performance as predictors of tumor treatment response and patient survival in patients with non-small cell lung cancer receiving chemoradiotherapy. AJR. 2012;198:75-82. 